\title{
Nanoparticle-mediated dual delivery of resveratrol and DAP5 ameliorates kidney ischemia/reperfusion injury by inhibiting cell apoptosis and inflammation
}

\author{
Yong $\mathrm{Xu}^{1, *}$, Bo Zhang ${ }^{2, *}$, Da Xie ${ }^{3, *}$, Yu Hu${ }^{1}$, Hai-Lun Li ${ }^{1}$, Li-Li Zhong ${ }^{1}$, Hong-Wu \\ Wang $^{1}$, Wei Jiang ${ }^{1}$, Zun-Ping Ke ${ }^{4}$ and Dong-Hui Zheng ${ }^{1}$ \\ ${ }^{1}$ Department of Nephrology, The Affiliated Huai'an Hospital of Xuzhou Medical University and The Second People's Hospital \\ of Huai'an, Huai'an, China \\ ${ }^{2}$ Department of Dermatology, Hubei Provincial Hospital of TCM, Hongshan, Wuhan, China \\ ${ }^{3}$ Department of Nephrology, The Secend Affiliated Hospital of Nanjing Medical University, Nanjing, China \\ ${ }^{4}$ Department of Cardiology, The Fifth People's Hospital of Shanghai, Fudan University, Shanghai, China \\ *These authors have contributed equally to this study and share first authorship \\ Correspondence to: Dong-Hui Zheng, email: zddwjj@126.com \\ Zun-Ping Ke, email: kzprenmin@163.com \\ Keywords: Res-DAP5 nanoparticles, apoptosis, inflammation, ischemia-reperfusion, acute renal injury \\ Received: December 01,2016 Accepted: February 12, $2017 \quad$ Published: April 17, 2017 \\ Copyright: $\mathrm{Xu}$ et al. This is an open-access article distributed under the terms of the Creative Commons Attribution License (CC-BY), \\ which permits unrestricted use, distribution, and reproduction in any medium, provided the original author and source are credited.
}

\section{ABSTRACT}

Ischemia reperfusion ( $I / R)$ injury is a leading cause of acute kidney injury with high morbidity and mortality due to limited therapy. NMDA receptor inhibitor (DAP5) and resveratrol (Res) could ameliorate kidney I/R injury, but their use is limited by low aqueous solubility and poor stability. Here, we examined the potential protective effects of Res-DAP5 nanoparticles (NP) against renal I/R injury. Mice were subjected to renal ischemia for $\mathbf{3 0} \mathrm{min}$ followed by reperfusion for $\mathbf{2 4} \mathrm{h}$. The results showed that Res-DAP5-NP could decreased serum creatinine ( $\mathrm{Cr}$ ) and urea nitrogen (BUN), alleviated tubular damage and oxidative stress. In addition, Res-DAP5-NP suppressed cell apoptosis, promoted the expression of p-DAPK, and inhibited the expression of p-CaMK and p-AKT. Furthermore, Res-DAP5-NP decreased the production of proinflammatory cytokines such as tumor necrosis factor-a, IL-1 $\beta$, IL-6, and p-IKBa induced by renal I/R injury. In addition, Res-DAP5-NP also attenuated renal I/R injury in vivo, as manifested by increase in cell viability, SOD level, and the expression of p-DAPK, decreases in intracellular $\mathrm{Ca}^{2+}$ concentration and the expression of $\mathrm{p}-\mathrm{CaMK}$. Taken together, our findings indicates that Res-DAP5-NP could effectively protect renal I/R injury by inhibiting apoptosis and inflammation responses, possibly through AKT/NMDA/CaMK/DAPK and NF-KB pathways.

\section{INTRODUCTION}

Acute kidney injury incuced by ischemia/ reperfusion (I/R) is a pivotal cause of acute renal failure with high morbidity and mortality [1]. However, there is limited therapy in clinical applications presently. Several pathogenesis cause to renal I/R injury including excitatory toxicity, intracellular $\mathrm{Ca} 2+$ overload, oxidative stress, etc. Nevertheless, the precise mechanisms are not well understood. It has been reported that cell apoptosis and inflammation serves a key function in acute kidney injury incuced by $\mathrm{I} / \mathrm{R}[2,3]$. Therefore, inhibiting apoptosis and inflammation may be an effective strategy to attenuate renal I/R injury.

$\mathrm{N}$-methyl-d-aspartate (NMDA) is excitatory neurotransmitter involved in learning and memory formation. Recently, the role of NMDA is also discovered in peripheral organs such as bones, cardiovascular, and kidneys [4]. It has been reported that hyperactivity of NMDA receptors in kidneys leads to renal damage in 
rodents [5]. Antagonism of allosteric sites of NMDA receptor demonstrated renoprotection against I/Rinduced acute kidney injury in rats [6]. Activation of NMDA receptor could cause calcium flux, activation of calcineurin, death-associated protein kinases (DAPK) dephosphorylation, and subsequent promote DAPK mediated death pathway, which is one of the main mechanisms of NMDA toxicity [7]. Kishino $M$ et al have found that $\mathrm{I} / \mathrm{R}$ stimuli enhanced DAPK catalytic activity, whereas DAPK deletion mutant attenuated kidney malfunction after I/R injury [8], which furtherly demonstrated the function of DAPK pathway in acute kidney injury incuced by $\mathrm{I} / \mathrm{R}$.

Oxidative stress has widely been recognized as one of the main pathogenesis in acute kidney injury incuced by I/R. Previous study showed that ROS could sensitize $\mathrm{Ca}^{2+} /$ calmodulin-dependent kinase (CaMK) to $\mathrm{Ca}^{2+}$, which is upstream signal of DAPK [9]. More importantly, it have been reported that in response to oxidative stimuli, CaMKII activation might only need extremely low $\mathrm{Ca} 2+/$ CaM levels [7]. So, we hypothesized that excessive ROS generated in the I/R-injured tissues might comprise the effect of NMDAR inhibitor, which is to say, cause NMDAR inhibitor "desensitization". Recent studies showed that combined therapy using agents with different molecular mechanisms achieved better therapeutic effects than single drug strategies and at least partially counteracted drug resistance $[10,11]$. Based on the above analysis, we proposed that NMDAR inhibitor combined antioxidants might serve as a new strategy for treatment of renal I/R injury.

Resveratrol (Res), a naturally occurring plantderived molecule, was shown to have potent antioxidant properties. It has been reported that Res as a scavenger of free radical might improve acute kidney injury induced by $\mathrm{I} / \mathrm{R}$ in rats [12]. However, in vivo studies on this regard have been limited by fast clearance and low bioavailability of Res [13]. Many studies have shown that nanoparticles can overcome these shortcomings to increase Res delivery [13-15]. Particularly, poly( $\epsilon$-caprolactone)-poly(ethylene glycol) (PCL-PEG) copolymer has been widely served for the delivery of anticancer agents because of its perfect properties $[16,17]$. Nevertheless, it is not known if PCL-PEG copolymers could serve for the delivery of kidney protectants. It is widely accepted that combined therapies against $I / R$ injury might be more effective than monotherapy, which because it could function through several different mechanisms [18]. So, our research attempted to investigate the role of Res and NMDAR inhibitor (DAP5) co-delivered with nanoparticles on kidney I/R injury and the underlying mechanisms.

\section{RESULTS}

\section{Characterization of Res-DAP5-NP}

As shown in Figure 1, the morphology of ResDAP5-NP was observed by transmission electron microscopy, with almost spherical shape at a size of around $200 \mathrm{~nm}$. The water solution of Res-DAP5-NP shows a yellow color and is basically transparent. The mean size of Res-DAP5-NP was about $152 \mathrm{~nm}$, while the surface charge of Res-DAP5-NP was slightly negative. Figure $1 \mathrm{D}$ shows a quick release of Res and DAP5, about $40 \%$, within the first 5 hours, which may have resulted from the inevitable affiliation of certain drugs to the surface of ResDAP5-NP. In the following process, Res and DAP5 was released in a controlled manner from the nanoparticles. It is clear that Res-DAP5-NP characterized by a sustained releasing pattern, could be a promising novel formulation of Res-DAP5-NP for future applications. We also found that there were obvious yellow-green fluorescence gathered in the renal $\mathrm{I} / \mathrm{R}$ area.

\section{Effects of Res-DAP5-NP on renal function}

As shown in Figure 2, kidney I/R injury cause a sharp raise in the contents of BUN and Cr. However, the levels of BUN and $\mathrm{Cr}$ in the Res, DAP5, Res+DAP5, and Res-DAP5-NP groups decreased significantly. In addition, the levels of BUN and Cr in Res-DAP5-NP group were obviously lower than those in the Res, DAP5, and Res+DAP5 groups. These data indicated that Res-DAP5NP could attenuate renal dysfunction following renal I/R injury.

\section{Effect of Res-DAP5-NP on renal histopathological changes}

As shown in Figure 3A, histological alterations such as tubular dilatation, cytoplasmic spaces, and cell necrosis were observed in the I/R group. However, compared to the $\mathrm{I} / \mathrm{R}$ group, there were less alterations in the Res, DAP5, Res+DAP5, and Res-DAP5-NP groups, with the least being in Res-DAP5-NP group. Similarly, as shown in Figure 3A by transmission electron microscopy, cells lost their normal morphology, and microvilli shed into the lumen following I/R injury. However, the Res, DAP5, Res+DAP5, and Res-DAP5-NP groups exhibited less damage to cellular morphology, with Res-DAP5-NP group being the least damaged. These results demonstrated that Res-DAP5-NP pretreatment could decrease renal pathological damage induced by I/R injury.

\section{Effects of Res-DAP5-NP on oxidative stress}

As shown in Figure 4, compared with the control group, the levels of ROS and MDA of renal tissues in the $I / R$ group markedly increased. Pretreatment with 
Res, DAP5, Res+DAP5, and Res-DAP5-NP significantly reduced the contents of ROS and MDA in kidney tissues. Moreover, the contents of ROS and MDA in Res-DAP5NP group were lower than those in the Res, DAP5, and Res+DAP5 groups. These data implied that Res-DAP5NP pretreatment can suppress oxidative stress induced by renal I/R injury.

\section{Effects of Res-DAP5-NP on cell apoptosis}

Apoptosis is another cause of renal injury following I/R. As shown in Figure 5, there were many apoptotic cells in the I/R group as indicated by TUNEL assay. On the contrary, Res, DAP5, Res+DAP5, and Res-DAP5-NP significantly reduced the amount of apoptotic cells. However, the apoptotic cells number in Res-DAP5-NP group was fewer than those in the Res,
DAP5, and Res+DAP5 groups. Furtherly, we found that caspase-3 activity increase markedly following $\mathrm{I} / \mathrm{R}$ injury. In contrast, the caspase-3 activity in the Res, DAP5, Res+DAP5, and Res-DAP5-NP groups following I/R was reduced, while the least in the ResDAP5-NP group. These data implied that Res-DAP5NP pretreatment suppressed apoptosis upon renal I/R injury.

\section{Effect of Res-DAP5-NP on expression of p-CaMK, p-DAPK, and p-AKT}

Western blot showed that comparing with the $\mathrm{I} / \mathrm{R}$ group, the expression levels of p-DAPK in the Res, DAP5, Res+DAP5, and Res-DAP5-NP groups remarkably increased, and were most in Res-DAP5-NP group. Interestingly, comparing with the $\mathrm{I} / \mathrm{R}$ group, the expression
A

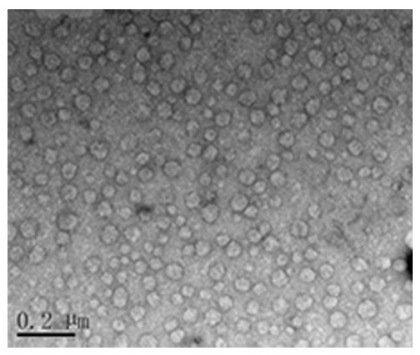

C

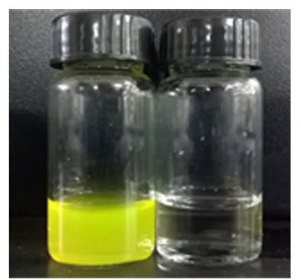

D

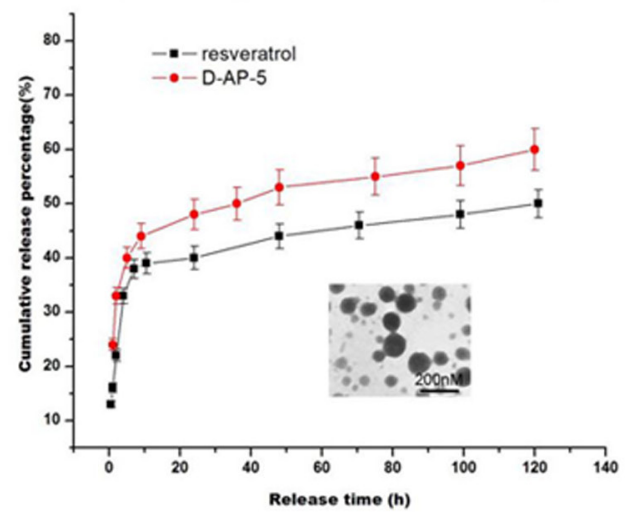

B
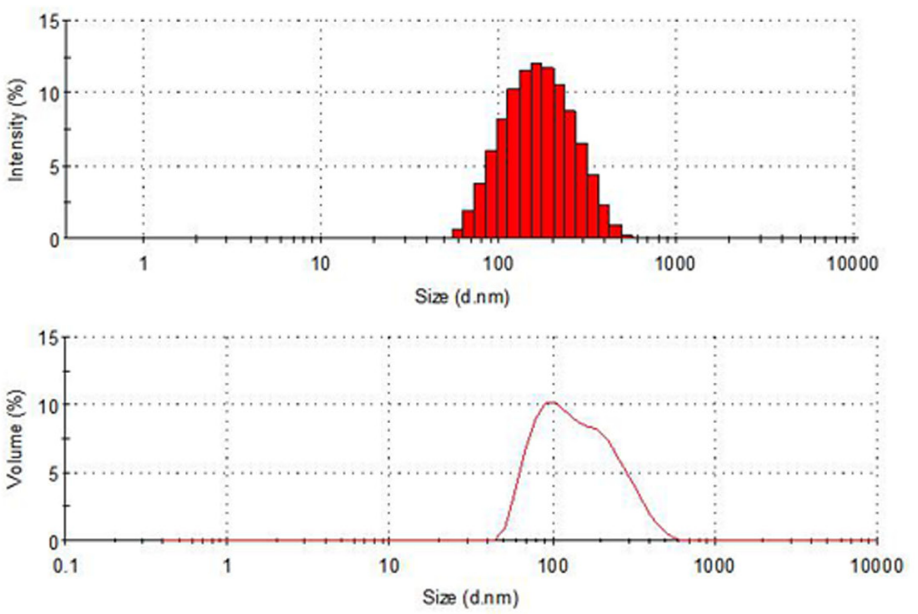

E
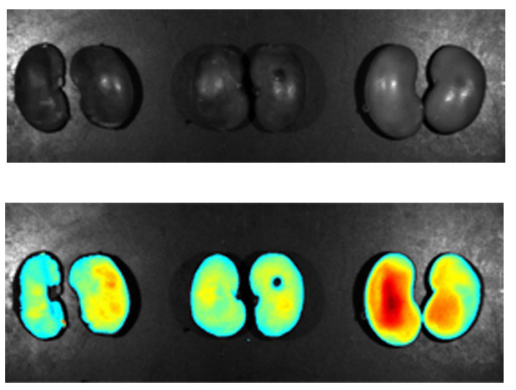

Figure 1: Characterization of Res-DAP5-NP. (A) Morphology of Res-DAP5-NP. (B) Size and Zeta potential of Res-DAP5-NP. (C) Pictures of deionized water and solutions of Res-DAP5-NP. (D) Cumulative in vitro release profile of Res and DAP5 from Res-DAP5-NP. (E) Res-DAP5-NP gathered in the renal ischemia-reperfusion area. Data are expressed as mean $\pm \operatorname{SD}(n=3)$. 

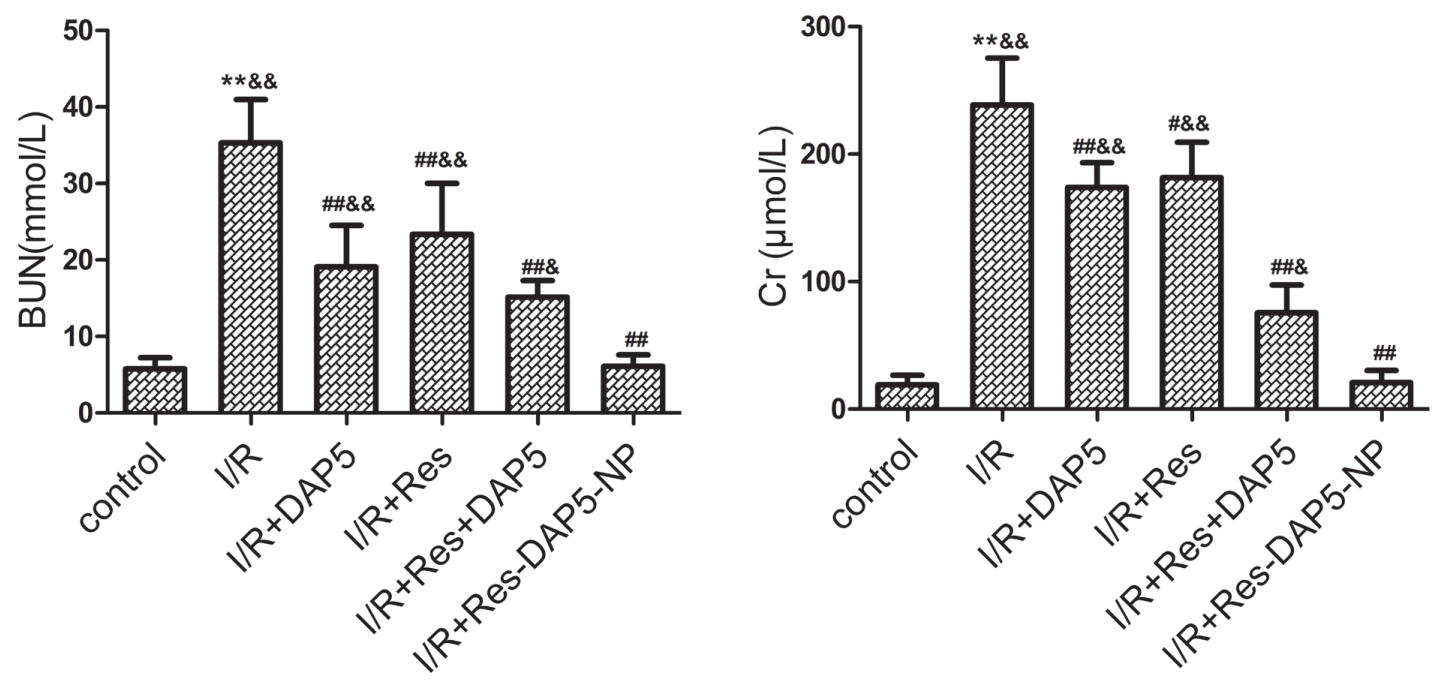

Figure 2: Effects of Res-DAP5-NP on serum levels of urea nitrogen (BUN) and Creatinine (Cr) in rats induced by renal I/R injury. Data are expressed as mean $\pm \mathrm{SD}(\mathrm{n}=3) .{ }^{* *} P<0.01$ vs the control group; ${ }^{\sharp} P<0.05,{ }^{\# \#} P<0.01$ vs the I/R group; ${ }^{\&} P<0.05$, $\& \& P<0.01$ vs the Res-DAP5-NP group.

A

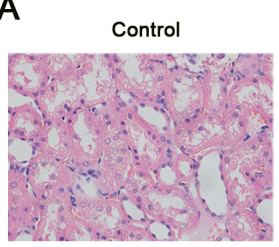

I/R+Res

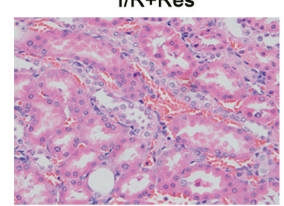

C

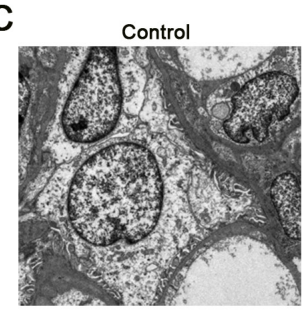

I/R+Res

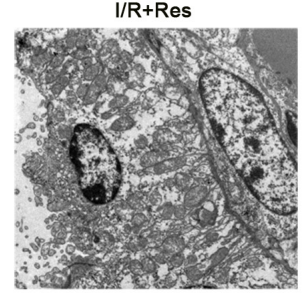

I/R

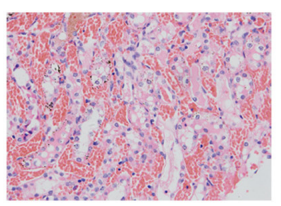

I/R+Res+DAP5
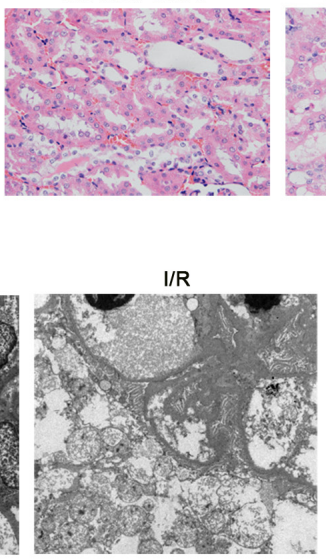

I/R+Res+DAP5

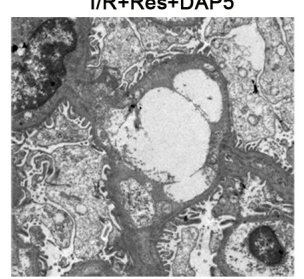

B

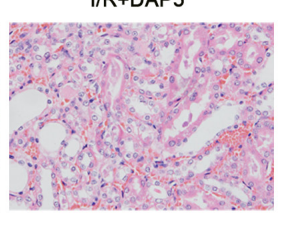

I/R+Res-DAP5-NP
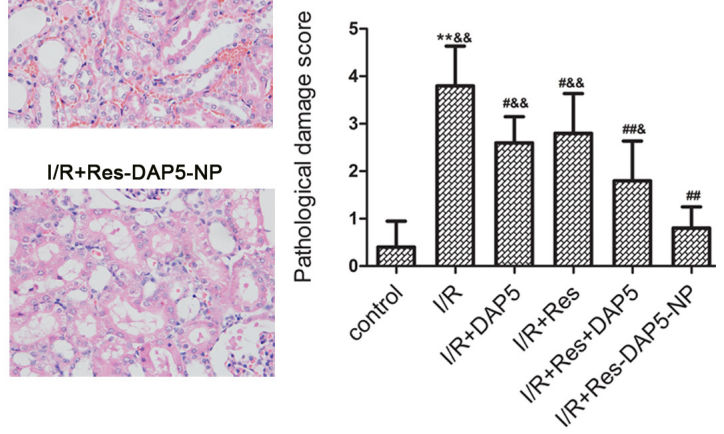

I/R+DAP5

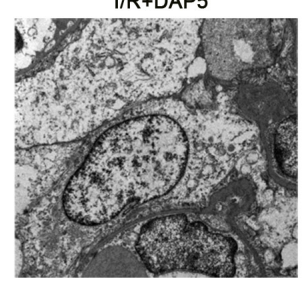

I/R+Res-DAP5-NP

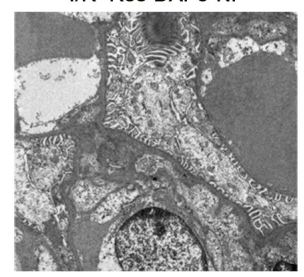

Figure 3: Histopathologic evaluation of kidney I/R injury. (A) Light microscope images $(\times 400)$. Semi-quantitative injury scores, expressed as the mean $\pm \mathrm{SD}(\mathrm{n}=5)$, are shown in $(\mathbf{B}){ }^{* *} P<0.01$ vs the control group; ${ }^{*} P<0.05,{ }^{\# \#} P<0.01$ vs the I/R group; ${ }^{*} P<0.05$, \&\& $P<0.01$ vs the Res-DAP5-NP group. (C) Transmission electron microscopy analysis of the effect of Res-DAP5-NP on renal I/R injury. Micrographs shown were taken at $\times 10,000$ magnification. 
levels of p-CaMK and p-AKT were lower in the Res, DAP5, Res+DAP5, and Res-DAP5-NP groups obviously reduced, while were least in Res-DAP5-NP group (Figure 6).

\section{Effect of Res-DAP5-NP on inflammatory factor}

As shown in Figure 7A, I/R injury can induce an obvious increase in TNF- $\alpha$, IL-1 $\beta$, and IL-6. Comparing with the I/R group, TNF- $\alpha$, IL- $1 \beta$, and IL- 6 in Res, DAP5, Res+DAP5, and Res-DAP5-NP groups decreased significantly. Furthermore, the expression levels of these factors in Res-DAP5-NP group were obviously less than those in the Res, DAP5, and Res+DAP5 groups.

$\mathrm{NF}-\kappa \mathrm{B}$ is a well-known regulator of the transcription of inflammatory gene including TNF- $\alpha$, IL- $1 \beta$, and IL-6 during acute kidney injury. We also studyed the phosphorylation of I $\mathrm{B} \alpha$. Upon exposure to Res-DAP5$\mathrm{NP}$, the $\mathrm{p}-\mathrm{I} \kappa \mathrm{B} \alpha$ was significantly reduced. These results revealed that Res-DAP5-NP suppressed inflammatory response induced by kidney I/R injury.

\section{Effect of Res-DAP5-NP on renal H/R injury in vitro}

As shown in Figure $8 \mathrm{~A}, \mathrm{H} / \mathrm{R}$ decreased cell viability, which was reversed to some degree by ResDAP5-NP $(10 \mu \mathrm{M})$. Furthermore, the activities of SOD significantly increased by Res-DAP5-NP compared with that in the H/R group (Figure 8B). Consistent with the results on enzymes, Res-DAP5-NP significantly reduced the intracellular $\mathrm{Ca}^{2+}$ concentration compared with that in the H/R group by fluo-3/AM (Figure 8C). To determine whether the CaMK-DAPK pathway was activated by Res-DAP5-NP, we measured the phosphorylated CaMK and DAPK, the results of which showed that the p-DAPK level increased remarkably and the p-CaMK level reduced significantly in the Res-DAP5-NP group (Figure 8D). Our findings as a whole lend to the conclusion that Res-DAP5-NP could prevent ischemia-reperfusion acute kidney injury in vitro.

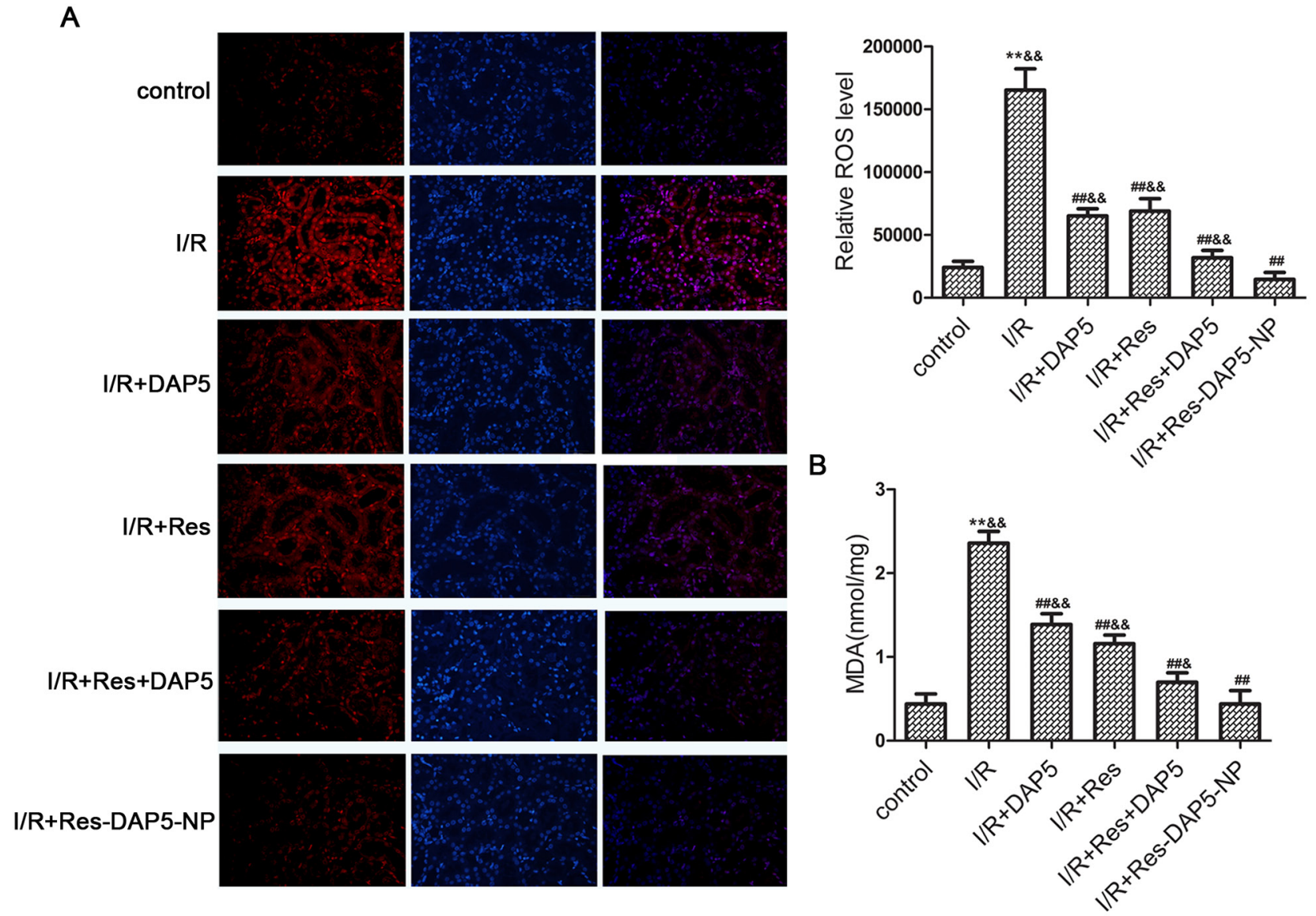

Figure 4: Effects of Res-DAP5-NP on oxidative stress in rats induced by renal I/R injury. (A) The ROS generation. Data are expressed as mean $\pm \mathrm{SD}(\mathrm{n}=3)$. (B) The level of MDA contents. ${ }^{* *} P<0.01$ vs the control group; ${ }^{\#} P<0.05,{ }^{\#} P<0.01 \mathrm{vs}$ the I/R group; ${ }^{\&} P<0.05,{ }^{\&} P<0.01$ vs the Res-DAP5-NP group. 


\section{DISCUSSION}

Apoptosis and inflammation were reported as crucial mechanisms underlying kidney I/R injury, and thus potential therapeutics against renal injury $[2,3]$.

Res and DAP5 are potent kidney protectants through many mechanisms to alleviate $\mathrm{I} / \mathrm{R}$ injury. However, their efficacies are limited due to poor water solubility and challengings in target delivery. Previous studies have shown that nanoparticles can overcome these shortcomings [16]. In this study, we constructed codelivered DAP5 and Res using PCL-PEG nanoparticles and aimed at investigating the effects of Res-DAP5-NP on renal I/R injury.

In our research, we firstly observed that ResDAP5-NP significantly decreased kidney dysfunction and histopathologic changes following $\mathrm{I} / \mathrm{R}$, demonstrating the protection of Res-DAP5-NP in acute kidney injury.

To explore the underlying mechanisms, we examined the influence of Res-DAP5-NP on oxidative

A
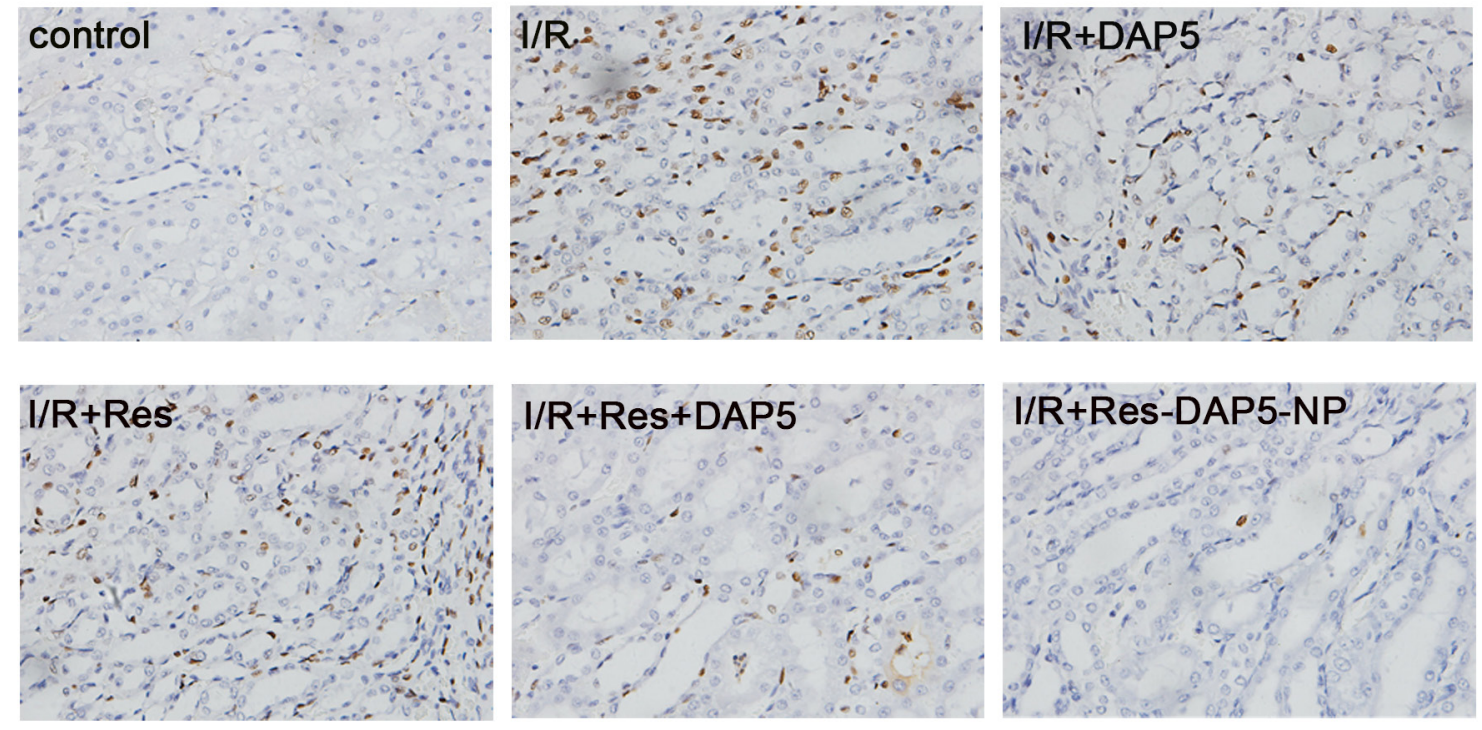

B

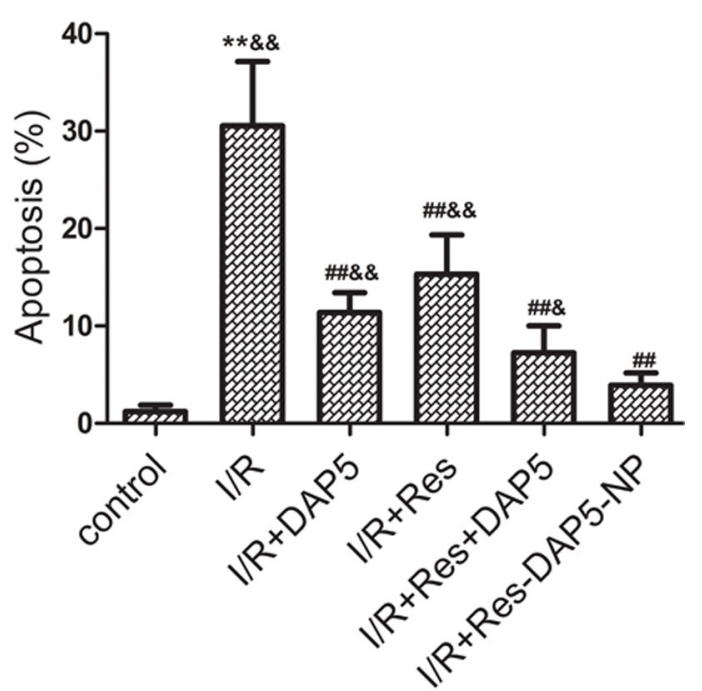

C

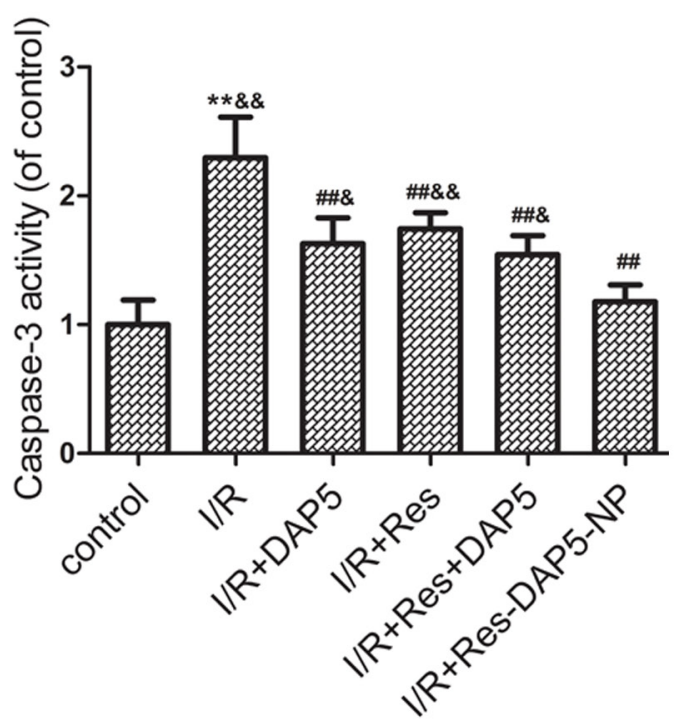

Figure 5: Effects of Res-DAP5-NP on cell apoptosis induced by renal I/R injury. (A) Representative images (magnification, $\times 400$ ) of renal TUNEL assay from rats subjected to renal I/R. (B) The percentage of TUNEL-positive cells in the kidney sections increased following I/R. (C) Effects of Res-DAP5-NP on caspase 3 activity in kidney tissues after I/R injury, quantitatively estimated as the foldchange relative to the I/R group. Data are expressed as mean $\pm \mathrm{SD}(\mathrm{n}=3) .{ }^{* *} P<0.01$ vs the control group; ${ }^{\#} P<0.05$, ${ }^{\#} P<0.01$ vs the I/R group; ${ }^{\&} P<0.05,{ }^{\&} P<0.01$ vs the Res-DAP5-NP group. 
stress. In this study, we demonstrated that Res-DAP5-NP reversed the elevated MDA content and ROS accumulation in vivo, and recovered the levels of SOD in vitro upon renal I/R injury, suggesting that Res-DAP5-NP attenuate acute kidney injury induced by $\mathrm{I} / \mathrm{R}$ possibly through its antioxidant properties.
Apoptosis is a vital pathological processes in acute kidney injury induced by $\mathrm{I} / \mathrm{R}[2,3]$, which was significantly inhibited by Res-DAP5-NP. In addition, Res-DAP5-NP promoted the expression of p-DAPK and inhibited the expression of $\mathrm{p}-\mathrm{CaMK}$ and $\mathrm{p}-\mathrm{AKT}$ in vivo. Furthermore, Res-DAP5-NP also attenuated renal I/R
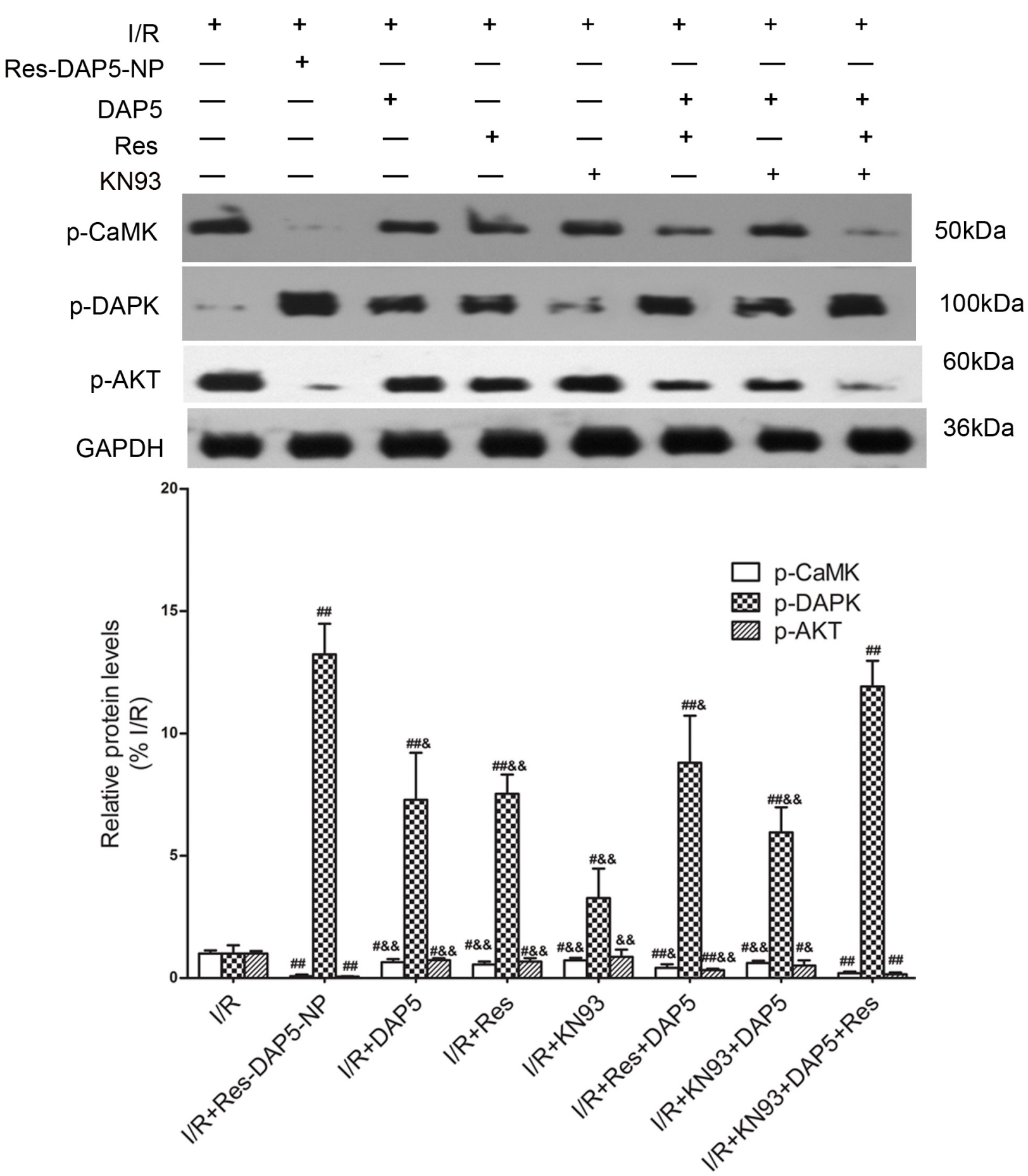

Figure 6: Effects of Res-DAP5-NP on expression of p-CaMK, p-DAPK, and p-AKT in rats induced by renal I/R injury. Data are expressed as mean $\pm \mathrm{SD}(\mathrm{n}=3)$. ${ }^{\#} P<0.05,{ }^{\#} P<0.01$ vs the I/R group; ${ }^{\&} P<0.05$, \&\& $P<0.01$ vs the Res-DAP5-NP group. 
A

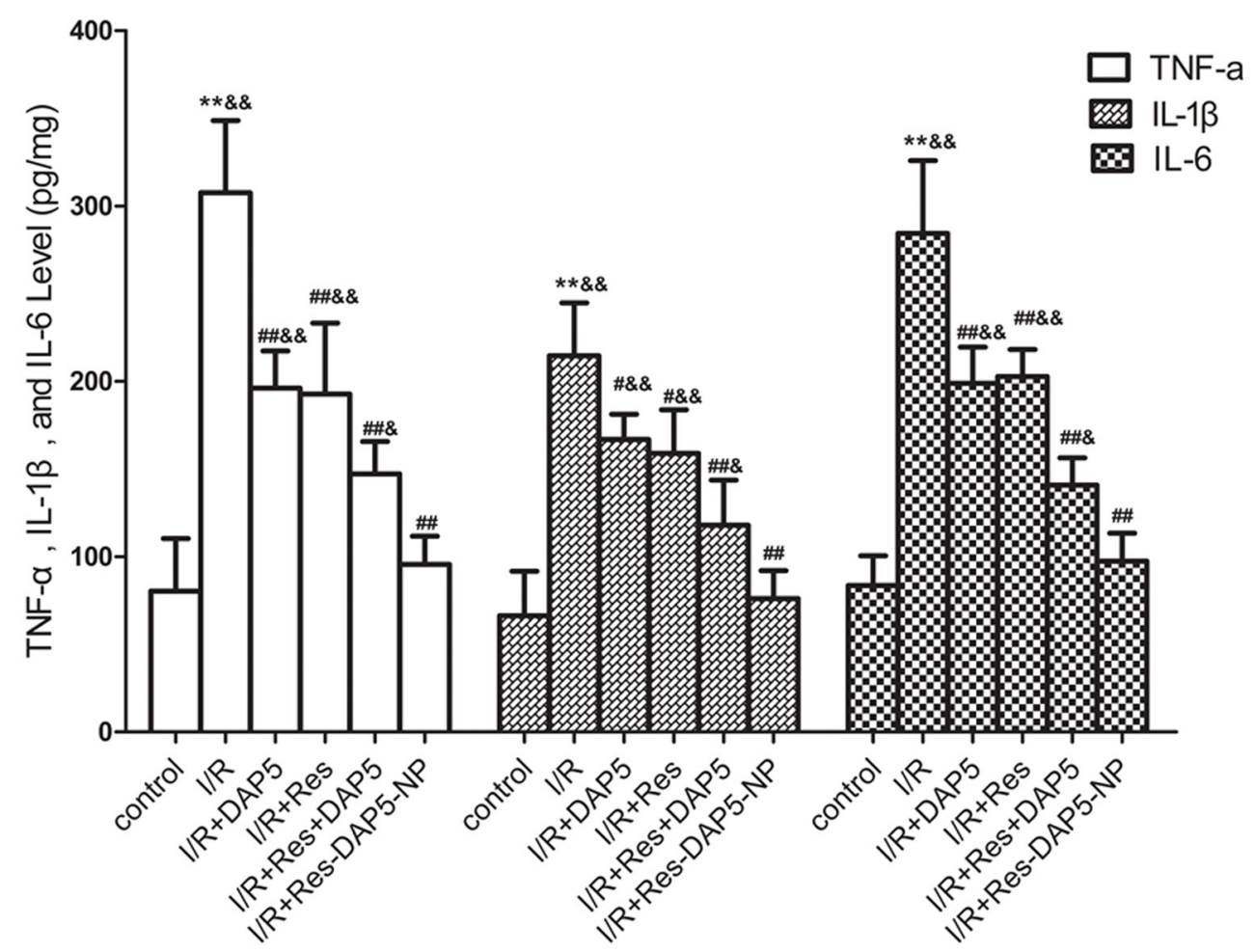

B

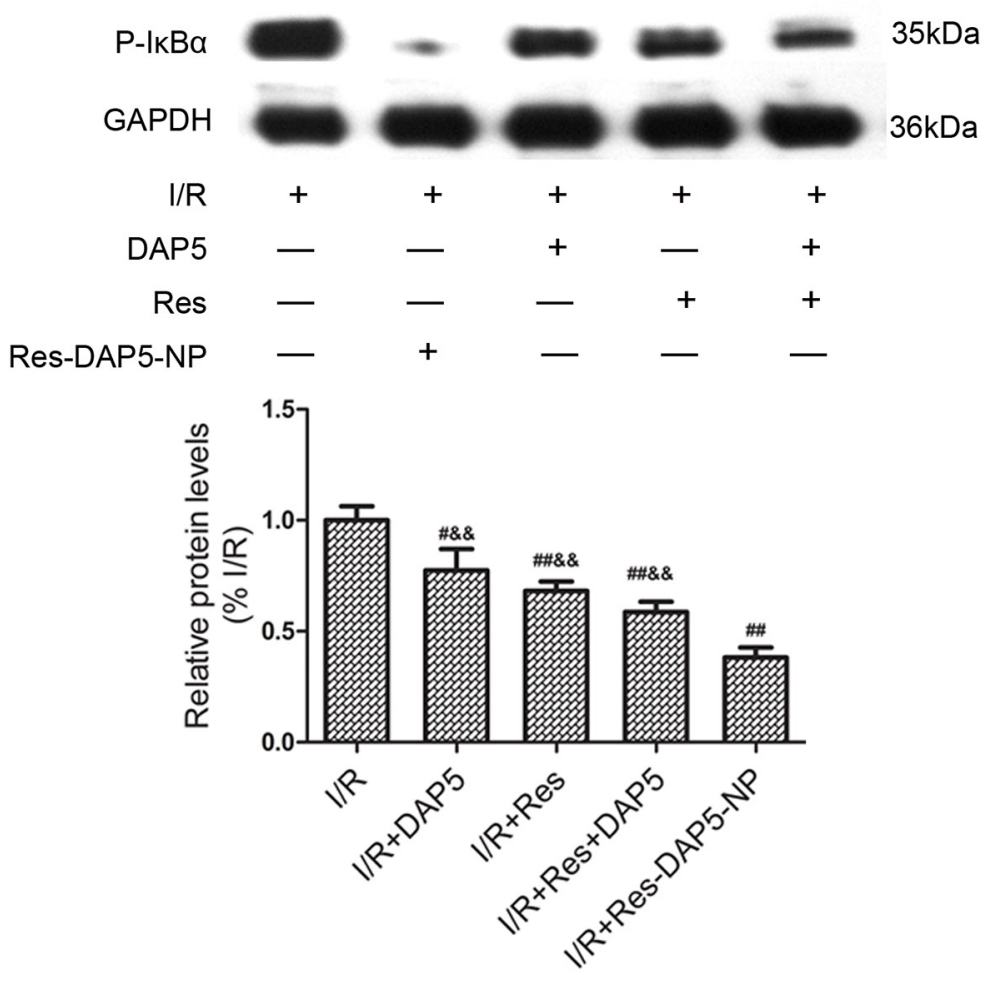

Figure 7: Effects of Res-DAP5-NP on pro-inflammatory factors (A) and phosphorylation of IKB $\alpha(B)$ in rats induced by renal I/R injury. Data are expressed as mean $\pm \mathrm{SD}(\mathrm{n}=3) .{ }^{* *} P<0.01$ vs the control group; ${ }^{*} P<0.05$, ${ }^{\#} P<0.01$ vs the I/R group; ${ }^{\&} P<0.05$, \&\& $P<0.01$ vs the Res-DAP5-NP group. 
injury in vitro, manifested by increased cell viability, SOD level, and expression of p-DAPK, reduced intracellular $\mathrm{Ca}^{2+}$ concentration and expression of $\mathrm{p}-\mathrm{CaMK}$. Taken together, our findings indicates that Res-DAP5-NP could effectively protect acute kidney injury induced by $\mathrm{I} / \mathrm{R}$ through AKT/NMDA/CaMK/DAPK signaling.

There is growing evidence that inflammation erves a key function in acute kidney injury induced by $\mathrm{I} / \mathrm{R}$ $[2,3,20]$. Our research demonstrated that Res-DAP5NP decreased the levels of TNF- $\alpha$, IL-1 $\beta$, and IL- 6 in renal tissue and represents a feasible method to reduce inflammation-related injury. Furthermore, NF- $\kappa \mathrm{B}$ is also a well-known regulator of the transcription of inflammatory gene during acute kidney injury [21]. Herein, we demonstrated that NF- $\kappa \mathrm{B}$ involved in inflammationrelated injury. These results suggest that Res-DAP5-NP could suppressed inflammatory response induced by kidney I/R injury via inhibiting activation of NF- $\kappa B$.

In conclusion, our research showed that Res-DAP5NP can protect rats against acute kidney injury induced by $\mathrm{I} / \mathrm{R}$ through its anti-apoptosis and anti-inflammatory activities, possibly via AKT/NMDA/CaMK/DAPK and
A

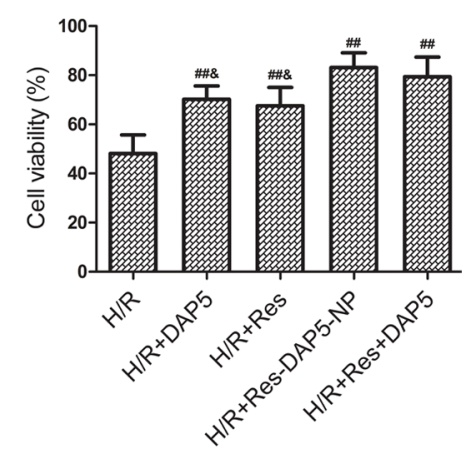

B

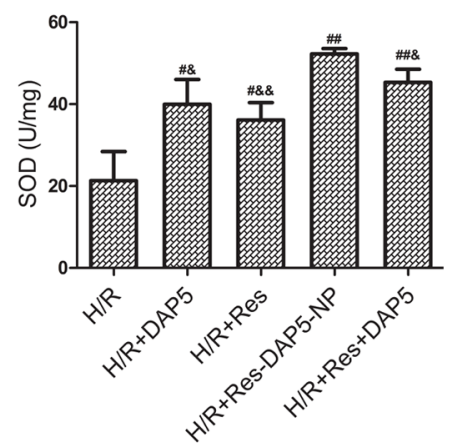

$\mathrm{C}$

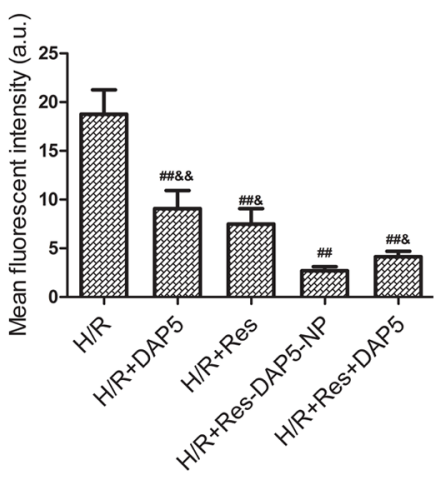

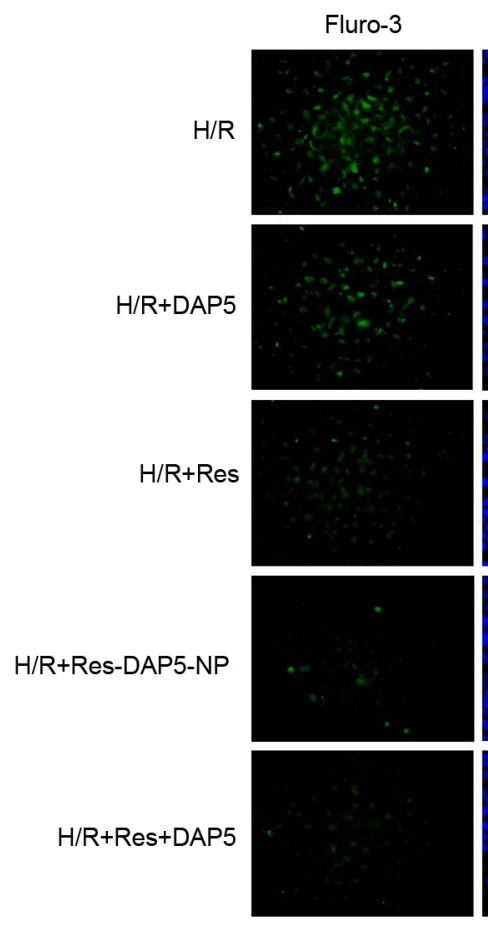
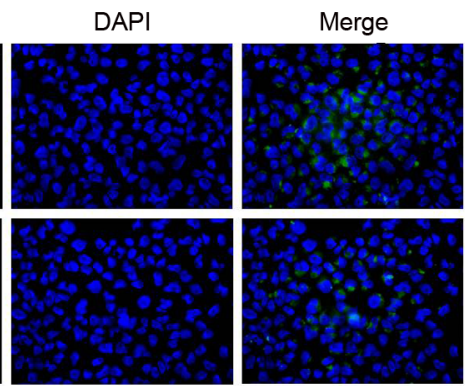

D
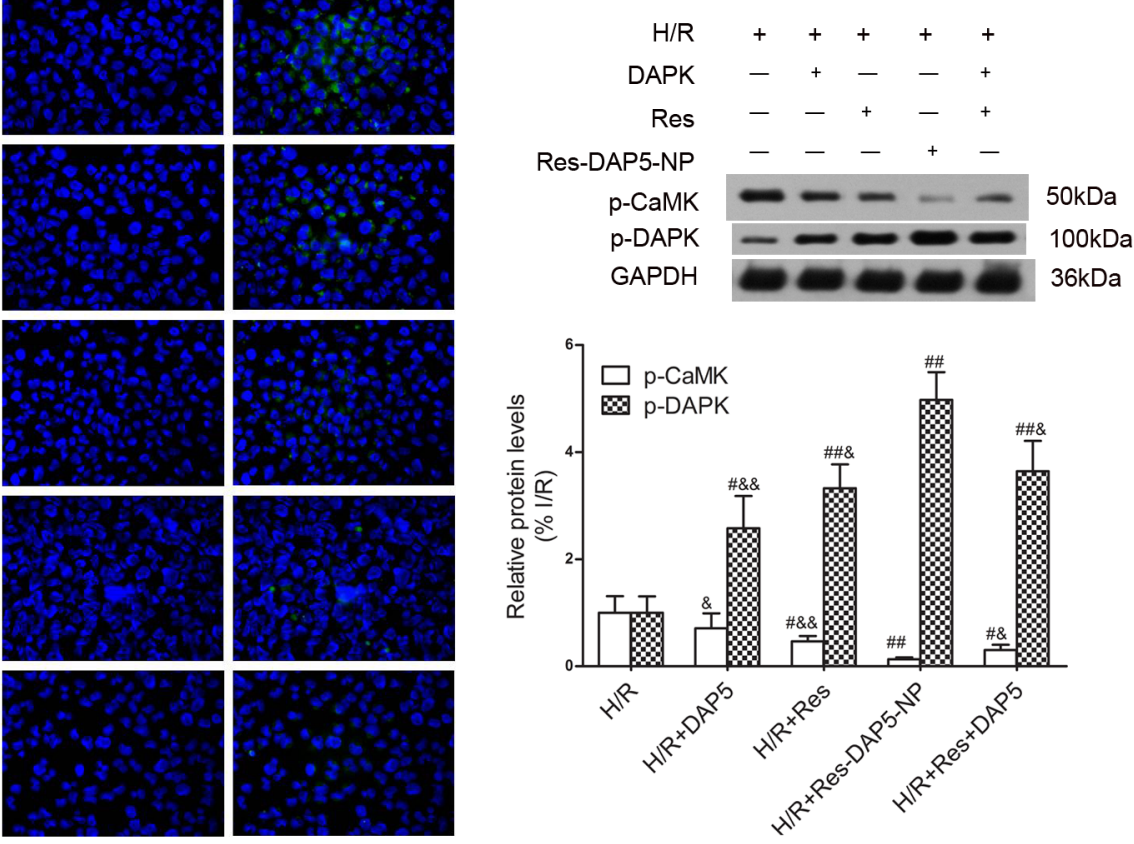

Figure 8: Effect of Res-DAP5-NP on renal I/R injury in vitro. (A) cell viability was assessed by MTT assay. (B) Effects of ResDAP5-NP on SOD levels after renal I/R injury. (C) Effect of Res-DAP5-NP on fluorescence intensity of intracellular Ca ${ }^{2+}$ after renal I/R injury. (D) Effects of Res-DAP5-NP on expression of p-CaMK and p-DAPK after renal I/R injury. Data are expressed as mean \pm SD $(n=3)$. ${ }^{\#} P<0.05,{ }^{\# \#} P<0.01$ vs the I/R group; ${ }^{\&} P<0.05$, \& \& $P<0.01$ vs the Res-DAP5-NP group. 
NF- $\kappa$ B pathway. Further studies in human clinical trials are necessary to exploit Res-DAP5-NP as a possible drug for the prevention of acute kidney injury.

\section{MATERIALS AND METHODS}

\section{Construction of Res-DAP5 nanoparticles (Res-DAP5-NP)}

Res-DAP5-NP were prepared as follows. Briefly, an amount of Res, DAP5 and PVP-b-PCL diblock copolymer were dissolved in acetone. The obtained organic solution was added dropwise into distilled water (ten times the volume) under moderate stirring at $25^{\circ} \mathrm{C}$. Subsequently, the solution was dialyzed in a dialysis bag to thoroughly remove the acetone. Finally, solutions of drug-loaded nanoparticles was lyophilized for further utilization. Green fluorescent coumarin-6 was incorporated into the NPs to visualize the uptake by cells.

\section{Animals}

Male Sprague-Dawley rats, aged 8-10 weeks (290$300 \mathrm{~g}$ ), were obtained from HFK Bio-Technology Co. Ltd. (Beijing, China). Animals were housed in a $12 \mathrm{~h}$ dark/ light cycle animals facility with controlled temperature and humidity for at least 1 week. All procedures involving animal use were in accordance with the guidelines for care and use of experimental animals and were approved by the institutional ethics committee at Huai' an Second Hospital Affiliated to Xuzhou Medical College.

\section{In vivo renal $\mathrm{I} / \mathrm{R}$ injury model}

The rat model of renal $\mathrm{I} / \mathrm{R}$ injury was induced by procedures similar to those described previously [19]. Briefly, rats were anesthetized with intraperitoneal injections of pentobarbital $(50 \mathrm{mg} / \mathrm{kg})$ and a midline abdominal incision was made. Renal ischemia was performed by clamping both renal vessels for $30 \mathrm{~min}$. Then, the clamps were removed to reinitiate renal blood flow. Sham-operated rats underwent the same surgical procedure without clamping. After $24 \mathrm{hrs}$ recovery, rats were sacrificed, blood and tissue were harvested for analysis. To determine the effect of drugs, rats were treated with Res (2 mg/kg), DAP5(a NMDAR inhibitor, $2 \mathrm{mg} / \mathrm{kg}$ ), KN93(a CaMKII inhibitor, $1 \mathrm{mg} / \mathrm{kg}$ ) and Res-DAP5 nanoparticles $(2 \mathrm{mg} / \mathrm{kg}$ ) by tail vein injection $3 \mathrm{~h}$ before I/R surgery.

\section{Assessment of renal function}

After reperfusion for $24 \mathrm{~h}$, blood samples were collected. Serum creatinine $(\mathrm{Cr})$ and blood urea nitrogen (BUN) levels were measured using commercially available kits (Jiancheng Pharmaceuticals, Nanjing, China) according to the manufacturer's instructions.

\section{Histological analysis}

One-half of the removed renal tissue were fixed in $4 \%$ paraformaldehyde, embedded with paraffin, and sectioned into slides $(4 \mu \mathrm{m})$. Then, the renal sections were subjected to hematoxylin-eosin (HE) staining for histological evaluation. The histopathological changes were evaluated by a pathologist in a blinded fashion according to the degree of tubular necrosis, hemorrhage and cast formation, as follows: 0 , no change; 1 , change affecting less than $25 \%$ of the field; 2 , change affecting $25-50 \%$ of the field; 3 , change affecting $50-75 \%$ of the field; 4 , change affecting more than $75 \%$ of the field [19]. The other half of kidney samples were also observed by transmission electron microscope (Hitachi, Tokyo, Japan) under uranyl acetate and lead citrate double staining.

\section{Assays for ROS accumulation}

The fluorescent probe 2' 7'-dichlorodihydrofluorescein diacetate (DCFH-DA) was used to monitor the intracellular accumulation of ROS. Fresh frozen sections were incubated with $10 \mu \mathrm{M}$ DCFH-DA for $25 \mathrm{~min}$ at $37^{\circ} \mathrm{C}$ in the dark. After rinsed two times with PBS, the sections were subjected to fluorescence analysis under a fluorescence microscope (Olympus, Japan). The mean densities of fluorescent images were quantified with Image Pro Plus software (Media Cybernetics).

\section{Detection of SOD and MDA content}

In order to estimate the anti-oxidative activities of Res-DAP5-NP, the levels of SOD and MDA were determined by commercial available kits from Nanjing Jiancheng Bioengineering according to manufacturer's protocols.

\section{TUNEL assay}

Renal samples were fixed in 4\% paraformaldehyde, embedded with paraffin, and sectioned into slides $(4 \mu \mathrm{m})$. Apoptosis was detected by using the TUNEL kit (Roche Diagnostics, Annheim, Germany) in accordance with the kit protocols. TUNEL slides were photographed with a microscope and the percentage of TUNEL-positive cells were counted at a magnification of $\times 400$ for 5 fields per section.

\section{Caspase- 3 activity assay}

Renal tissue was homogenized in lysis buffer. Then, reaction buffer and caspase- 3 substrate peptide were mixed in 96 -well plates at $37^{\circ} \mathrm{C}$ for $4 \mathrm{~h}$ in the dark. After incubation, a microplate reader (excitation/emission $360 / 460 \mathrm{~nm}$ ) was used to analyzethe samples. 


\section{ELISA assay}

The protein levels of tumor necrosis factor- $\alpha$ (TNF- $\alpha$ ), interleukin (IL)-1 $\beta$, and IL-6 in plasma were measured by ELISA according to the manufacturer's instructions (Biosource International Inc, United States).

\section{Cell culture and hypoxia/reoxygenation $(\mathrm{H} / \mathrm{R})$ model}

Human proximal tubular cells (HK-2) were purchased by the Cell Bank of the Chinese Academy of Sciences (Shanghai, China). Cells were grown in DMEM supplemented with $10 \%$ fetal bovine serum at $37{ }^{\circ} \mathrm{C}$ in a 5\% CO2 incubator. For hypoxic treatment, HK-2 cells were incubated for $8 \mathrm{~h}$ in a atmosphere containing $1 \% \mathrm{O} 2$, $5 \% \mathrm{CO} 2$ and $94 \% \mathrm{~N} 2$, followed by reoxygenation $(95 \%$ $\mathrm{O} 2,5 \% \mathrm{CO} 2$,) for $24 \mathrm{~h}$.

\section{Cell viability assay}

MTT assy was used to measure cell viability. Briefly, HK-2 cells were seeded in 96 -well plates at $5 \times 10^{3}$ cells per well and incubated overnight to achieve $70 \%$ confluency. Next, cells were incubated with indicated drugs for $2 \mathrm{~h}$ prior to exposure to $\mathrm{H} / \mathrm{R}$ injury. After drug treatments, $5 \mathrm{mg} / \mathrm{ml}$ MTT solution was added, and incubated for $4 \mathrm{~h}$. Then, the medium was removed, and the dark blue crystals were dissolved in $150 \mu \mathrm{l}$ DMSO. The absorbance at $570 \mathrm{~nm}$ was measured.

\section{Measurement of intracellular calcium fluorescence intensity}

HK-2 cells were incubated with $4 \mu \mathrm{M}$ Fluo-3/AM in the dark for $40 \mathrm{~min}$ at $37^{\circ} \mathrm{C}$. After dyeing, the cells were washed three times with PBS. Then, the images of $\mathrm{Ca}^{2+}$ concentration were monitored by the confocal microscope (Olympus, LSM, Japan) and fluorescence intensity was detected using FV10-ASW software.

\section{Western blot}

Western blot analysis was performed as described previously [19]. In brief, protein samples were electrophoretically transferred onto a polyvinylidene fluoride membranes. Blots were blocked with $5 \%$ fatfree milk for $2 \mathrm{~h}$ at room temperature, incubated with primary antibodies against p-AKT (CST; 1:500), p-DAPK (Abcam; 1:500), p-CaMK (CST; 1:500), and IkB $\alpha$ (CST; $1: 500)$ at $4{ }^{\circ} \mathrm{C}$ overnight, and followed by incubation with HRP-linked secondary antibody for $1.5 \mathrm{~h}$. The results were analyzed by gray level of chemiluminescence.

\section{Statistics analysis}

Differences among groups were statistically tested by one-way analysis of variance (ANOVA) using SPSS 16.0. Statistical significance was defined as $P<0.05$. All experiments were repeated three times, and all data were expressed as mean \pm standard deviation (SD).

\section{ACKNOWLEDGMENTS}

This study was supported by the National Natural Science Fund of China (no. 81270817).

\section{CONFLICTS OF INTEREST}

The authors declare no conflict of interest.

\section{REFERENCES}

1. Tong F, Tang X, Li X, Xia W, Liu D. The effect of insulinloaded linear poly(ethylene glycol)-brush-like poly(l-lysine) block copolymer on renal ischemia/reperfusion-induced lung injury through downregulating hypoxia-inducible factor. Int J Nanomedicine. 2016; 11:1717-30.

2. Yang Z, Zhong Z, Li M, Xiong Y, Wang Y, Peng G, Ye Q. Hypothermic machine perfusion increases A20 expression which protects renal cells against ischemia/reperfusion injury by suppressing inflammation, apoptosis and necroptosis. Int J Mol Med. 2016; 38:161-71.

3. Liu XJ, Tan Y, Geng YQ, Wang Z, Ye JH, Yin XY, Fu B. Proximal tubule toll-like receptor 4 expression linked to inflammation and apoptosis following hypoxia/reoxygenation injury. Am J Nephrol. 2014; 39:337-47.

4. Singh AP, Singh N, Bedi PM. Pioglitazone ameliorates renal ischemia reperfusion injury through NMDA receptor antagonism in rats. Mol Cell Biochem. 2016; 417:111-18.

5. Lin CS, Hung SF, Huang HS, Ma MC. Blockade of the N-Methyl-D-Aspartate Glutamate Receptor Ameliorates Lipopolysaccharide-Induced Renal Insufficiency. PLoS One. 2015; 10:e0132204.

6. Pundir M, Arora S, Kaur T, Singh R, Singh AP. Effect of modulating the allosteric sites of N-methyl-D-aspartate receptors in ischemia-reperfusion induced acute kidney injury. J Surg Res. 2013; 183:668-77.

7. Palomeque J, Rueda OV, Sapia L, Valverde CA, Salas M, Petroff MV, Mattiazzi A. Angiotensin II-induced oxidative stress resets the $\mathrm{Ca} 2+$ dependence of $\mathrm{Ca} 2+$-calmodulin protein kinase II and promotes a death pathway conserved across different species. Circ Res. 2009; 105:1204-12.

8. Kishino M, Yukawa K, Hoshino K, Kimura A, Shirasawa N, Otani H, Tanaka T, Owada-Makabe K, Tsubota Y, Maeda M, Ichinose M, Takeda K, Akira S, Mune M. Deletion of the kinase domain in death-associated protein kinase attenuates tubular cell apoptosis in renal ischemia-reperfusion injury. J Am Soc Nephrol. 2004; 15:1826-34. 
9. Usui T, Okada M, Hara Y, Yamawaki H. Death-associated protein kinase 3 mediates vascular inflammation and development of hypertension in spontaneously hypertensive rats. Hypertension. 2012; 60:1031-39.

10. Bourgeois-Daigneault MC, St-Germain LE, Roy DG, Pelin A, Aitken AS, Arulanandam R, Falls T, Garcia V, Diallo JS, Bell JC. Combination of Paclitaxel and MG1 oncolytic virus as a successful strategy for breast cancer treatment. Breast Cancer Res. 2016; 18:83.

11. Deng Y, Yang M, Xu F, Zhang Q, Zhao Q, Yu H, Li D, Zhang G, Lu A, Cho K, Teng F, Wu P, Wang L, et al. Combined Salvianolic Acid B and Ginsenoside Rg1 Exerts Cardioprotection against Ischemia/Reperfusion Injury in Rats. PLoS One. 2015; 10:e0135435.

12. Sener G, Tuğtepe H, Yüksel M, Cetinel S, Gedik N, Yeğen BC. Resveratrol improves ischemia/reperfusioninduced oxidative renal injury in rats. Arch Med Res. 2006; $37: 822-29$.

13. Wang W, Zhang L, Le Y, Chen JF, Wang J, Yun J. Synergistic effect of PEGylated resveratrol on delivery of anticancer drugs. Int J Pharm. 2016; 498:134-41.

14. Kumar S, Lather V, Pandita D. A facile green approach to prepare core-shell hybrid PLGA nanoparticles for resveratrol delivery. Int J Biol Macromol. 2016; 84:380-84.

15. Lindner GR, Dalmolin LF, Khalil NM, Mainardes RM. Influence of the Formulation Parameters on the Particle Size and Encapsulation Efficiency of Resveratrol in PLA and PLA-PEG Blend Nanoparticles: A Factorial Design. J Nanosci Nanotechnol. 2015; 15:10173-82.

16. Shen Y, Leng M, Yu H, Zhang Q, Luo X, Gregersen H, Wang G, Liu X. Effect of amphiphilic PCL-PEG nanomicelles on HepG2 cell migration. Macromol Biosci. 2015; 15:372-84.

17. Gou M, Wei X, Men K, Wang B, Luo F, Zhao X, Wei Y, Qian Z. PCL/PEG copolymeric nanoparticles: potential nanoplatforms for anticancer agent delivery. Curr Drug Targets. 2011; 12:1131-50.

18. Hardy N, Viola HM, Johnstone VP, Clemons TD, Cserne Szappanos H, Singh R, Smith NM, Iyer KS, Hool LC. Nanoparticle-mediated dual delivery of an antioxidant and a peptide against the L-Type $\mathrm{Ca} 2+$ channel enables simultaneous reduction of cardiac ischemia-reperfusion injury. ACS Nano. 2015; 9:279-89.

19. Luo LN, Xie Q, Zhang XG, Jiang R. Osthole decreases renal ischemia-reperfusion injury by suppressing JAK2/STAT3 signaling activation. Exp Ther Med. 2016; 12:2009-14.

20. Ling H, Chen H, Wei M, Meng X, Yu Y, Xie K. The Effect of Autophagy on Inflammation Cytokines in Renal Ischemia/Reperfusion Injury. Inflammation. 2016; 39:347-56.

21. Xie DQ, Sun GY, Zhang XG, Gan H. Osthole Preconditioning Protects Rats Against Renal IschemiaReperfusion Injury. Transplant Proc. 2015; 47:1620-26. 